\title{
Galactooligosaccharide fibres exert immunomodulatory properties and interfere with riboflavin derivatives in an ex-vivo study
}

\author{
S. Del Fabbro ${ }^{1 *}$, P.C. Calder ${ }^{1,2,3}$ and C.E. Childs ${ }^{1,3}$ \\ ${ }^{1}$ School of Human Development and Health, Faculty of Medicine, University of Southampton, Southampton, UK, \\ ${ }^{2}$ NIHR Southampton Biomedical Research Centre, University Hospital Southampton NHS Foundation Trust and \\ University of Southampton, Southampton, UK and \\ ${ }^{3}$ Institute for Life Sciences, University of Southampton, Southampton, UK
}

Mucosal-associated invariant T (MAIT) cells are T lymphocytes with a key role in immune surveillance. Riboflavin derivatives produced by the gut microbiota are MAIT cell ligands ${ }^{(1)}$. Evidence suggests that unknown metabolites synthesised by probiotic strains modulate MAIT cell function ${ }^{(2,3)}$. Galactooligosaccharides (GOS) are prebiotics produced via transgalactosylation by $\beta$-galactosidases, which are expressed by gut bacteria ${ }^{(4)}$. We aim to assess whether GOS modulate the function of MAIT cells or other peripheral blood mononuclear cell (PBMC) subsets and whether cells respond differently to a riboflavin derivative in presence of GOS.

Healthy PBMCs $(\mathrm{n}=8)$ were cultured for $20 \mathrm{~h}$ with GOS (Bimuno $\left.{ }^{\circledR}\right)(12 \mathrm{mg} / \mathrm{mL})$, or a riboflavin derivative $(5-\mathrm{A}-\mathrm{RU} 0.18 \mu \mathrm{M}+$ methylglyoxal $1 \mu \mathrm{M}$ ), or co-stimulated with both. Unstimulated cells were used as control. One-way ANOVA or Kruskal-Wallis test followed by Bonferroni's or Dunn's post-hoc test were performed depending upon data distribution.

Treatment with GOS did not affect viability. PBMCs incubated with GOS presented lower CD4 expression on T helper cells (MFI $14,561 \pm 612.3$ vs $17,593 \pm 2,157 ; \mathrm{p}=0.0053)$ and secreted more IL-8 $(104.0 \pm 62.9 \mathrm{ng} / \mathrm{mL}$ vs $3.8 \pm 2.6 \mathrm{ng} / \mathrm{mL} ; \mathrm{p}=0.0005) \mathrm{compared}$ to control. Intracellular staining revealed that monocytes were responsible for the upregulated IL-8 expression. Co-treatment of cells with the riboflavin derivative and GOS resulted in decreased CD69 expression by lymphocytes (MFI 19,076 $\pm 2,648$ vs $15,761 \pm 1,158 \mathrm{p}=$ $0.0017)$, T cells (MFI 20,317 $\pm 2,303$ vs $16,572 \pm 1,439 \mathrm{p}<0.0001)$ and cytotoxic T cells $($ MFI 24,669 $\pm 4,249$ vs $20,188 \pm 3,485 \mathrm{p}=$ $0.0205)$ compared to riboflavin derivative alone, and in lower \% MAIT cells expressing IL-17A vs riboflavin derivative alone $(0.03 \pm$ 0.03 vs $0.49 \pm 0.40 \mathrm{p}=0.0008)$.

Overall, GOS showed immunomodulatory effects, including the modulation of CD4 expression and enhancement of IL-8 secretion. PBMCs responded differently to ligand challenge in presence of GOS, suggesting that the prebiotic may interfere with riboflavin metabolites or signal via similar pathways.

\author{
Acknowledgements \\ GOS (Bimuno®) was provided by Clasado Biosciences
}

\section{References}

1. Patel O, Kjer-Nielsen L, Le Nours J, et al. (2013) Nat Commun 4, 1-9.

2. Johansson MA, Bjorkander S, Mata Forsberg M, et al. (2016) Front Immunol 7, 1-15.

3. Hinks TS (2016) Immunology 148, 1-12.

4. Grimaldi R, Swann JR, Vulevic J, et al. (2016) Br J Nutr 116, 480-486. 